\title{
GRAPPA Trainees Symposium 2017: A Report from the GRAPPA 2017 Annual Meeting
}

\author{
Victoria Furer, Julia Manasson, Wolf-Henning Boehncke, and Christopher T. Ritchlin
}

\begin{abstract}
At the 2017 annual meeting of the Group for Research and Assessment of Psoriasis and Psoriatic Arthritis (GRAPPA) in Amsterdam, the Netherlands, a trainees symposium was held. Rheumatology and dermatology trainees engaged in psoriasis or psoriatic arthritis research presented their work. This report briefly reviews 6 oral presentations and 25 posters presented at the meeting. (J Rheumatol Suppl. 2018 June;94:4-10; doi:10.3899/jrheum.180130)
\end{abstract}

Key Indexing Terms: PSORIASIS DERMATOLOGIST

\author{
PSORIATIC ARTHRITIS \\ RHEUMATOLOGIST
}

GRAPPA

TRAINEE
The Group for Research and Assessment of Psoriasis and Psoriatic Arthritis (GRAPPA) held this year's trainees symposium at its 2017 annual meeting in Amsterdam, the Netherlands. Following the tradition of the past 7 years, rheumatology or dermatology trainees from North America, South America, and Europe who are current GRAPPA members or who were nominated by GRAPPA members were invited to submit abstracts based on recent research in psoriatic arthritis (PsA) or psoriasis $1,2,3,4,5,6,7$. A total of 40 abstracts were submitted and ranked by a committee of reviewers. Six trainees with the highest-scored abstracts were invited to deliver oral presentations, and 25 trainees presented posters that outlined key aspects of their research. Dr. Christopher T. Ritchlin (Rochester, New York, USA) and Dr. Wolf-Henning Boehncke (Geneva, Switzerland) co-chaired the symposium in which GRAPPA members discussed the findings presented by trainees and suggested how trainees might further develop their current research projects.

From the Tel Aviv Sourasky Medical Center, Sackler Faculty of Medicine, Tel Aviv University, Tel Aviv, Israel; Division of Rheumatology, Department of Medicine, New York University School of Medicine, New York; Division of Allergy, Immunology, and Rheumatology, University of Rochester Medical Center, Rochester, New York, USA; Department of Dermatology, Division of Dermatology and Venereology, Geneva University Hospital; Department of Pathology and Immunology, University of Geneva, Geneva, Switzerland.

As part of the supplement series GRAPPA 2017, this report was reviewed internally and approved by the Guest Editors for integrity, accuracy, and consistency with scientific and ethical standards.

V. Furer, MD, Tel Aviv Sourasky Medical Center, Sackler Faculty of Medicine, Tel Aviv University; J. Manasson, MD, Division of

Rheumatology, Department of Medicine, New York University School of Medicine; W.H. Boehncke, MD, Professor and Chair, Department of Dermatology, Division of Dermatology and Venereology, Geneva University Hospital, and Department of Pathology and Immunology, University of Geneva; C.T. Ritchlin, MD, MPH, Professor of Medicine, Division of Allergy, Immunology, and Rheumatology, University of Rochester Medical Center.

Address correspondence to Dr. V. Furer, Rheumatology Department, Tel Aviv Medical Center, Sackler School of Medicine, Weizmann St. 6, Tel Aviv-Yafo, Israel.E-mail:vikushf@gmail.com

\section{Oral Presentations}

A new and simpler tool for global psoriatic arthritis assessment: simplified Composite Psoriatic Disease Activity Index (Maria Laura Acosta Felquer, Buenos Aires, Argentina)

Psoriatic arthritis (PsA) is a heterogeneous disease with involvement of multiple domains. The Composite Psoriatic Disease Activity Index (CPDAI) is a comprehensive clinical tool accounting for 5 disease domains, including peripheral joints [joint count and Health Assessment Questionnaire (HAQ)], skin [Psoriasis Area and Severity Index (PASI) and Dermatology Life Quality Index], spine [Bath Ankylosing Spondylitis Disease Activity Index (BASDAI) and Ankylosing Spondylitis Quality of Life], enthesitis [Leeds Enthesitis Index (LEI) and HAQ], and dactylitis (digit count and HAQ). The CPDAI tool is a complex measure to perform in daily practice. Dr. Felquer's study evaluated the performance of a simplified CPDAI (sCPDAI) based on joint count, digit count, enthesitis (LEI) assessment, skin (body surface area) assessment, and patient-reported outcomes of HAQ and BASDAI in a large PsA cohort from the Measuring Outcome in Psoriatic Arthritis database.

The study included 214 consecutive PsA patients (111 women, $52 \%$ ) with a mean age of $49 \pm 12$ years. Seventy-six patients $(35.5 \%)$ achieved minimal disease activity (MDA). Median (interquartile range) Clinical Disease Activity Index (CDAI), clinical Disease Activity in Psoriatic Arthritis (cDAPSA), CPDAI, and PASI were 7 (4-16), 10 (5-18), 3 (2-5), and $0.8(0-3)$, respectively. A very good correlation was demonstrated between sCPDAI and most outcome measurements used in PsA [tender and swollen joint count, CPDAI, CDAI, cDAPSA, PASI, Psoriatic Arthritis Quality of Life, patient visual analog scale (VAS), and patient's and physician's global VAS]. Patients in MDA had significantly lower sCPDAI compared to patients not in MDA [mean (SD) 1.7 (1.4) vs 5.3 (2.8); p < 0.0001]. The sCPDAI area under the curve of the receiver-operating characteristic curve 
for MDA was 0.87 (95\% CI 0.83-0.92), with 4 as the best cutoff value to discriminate among patients not in MDA status (sensitivity: $68.42 \%$; specificity: $87.67 \%$; +LR: 5.55 , -LR: 0.36). The study results suggest that sCPDAI performed well in a large PsA cohort, had good correlation with most PsA measurement tools, and discriminated especially well among patients in MDA versus non-MDA.

Whole spine and sacroiliac joints magnetic resonance imaging of patients with psoriatic arthritis: descriptive study of the spine and SIJ involvement in a cross-sectional large cohort (Victoria Furer, Tel Aviv, Israel)

Detection of axial disease in psoriatic arthritis (PsA) has important implications. The prevalence and distribution of spinal changes in PsA as detected by magnetic resonance imaging (MRI) is largely unknown. Dr. Furer evaluated acute and structural changes in spine and sacroiliac joints (SIJ) by whole-spine MRI performed in a consecutive clinical cohort of PsA.

The study included 96 consecutively recruited patients with PsA with a mean age of $50 \pm 13$ years (50 male, 52\%), psoriasis/PsA duration of $19 \pm 13.6$ years/ $9 \pm 8$ years, Psoriasis Area and Severity Index (PASI) $3.9 \pm 8.9$, and Ankylosing Spondylitis Disease Activity Score with C-reactive protein $2.2 \pm 1$. Only $4.4 \%$ were carriers of the HLA-B27 antigen. The majority of patients (70\%) reported back pain, whereas inflammatory back pain (IBP) was present in $30 \%$. At the time of enrollment, $45 \%$ were treated with disease-modifying antirheumatic drugs and $35 \%$ with biologics. All patients underwent an MRI of the entire spine and SIJ. All MRI were evaluated by an experienced musculoskeletal radiologist blinded to the clinical data.

Active, structural, and total sacroiliitis were detected in $26 \%, 11.5 \%$, and $37.5 \%$ of patients, respectively. Spinal spondyloarthritis ( $\mathrm{SpA} ; \geq 3$ bone marrow edema segments or $\geq 4$ fatty vertebral corners) was demonstrated in $15.6 \%$ and involved the thoracic and lumbar segments, with a rare involvement of the cervical spine. Isolated spinal changes were detected in $2.1 \%$ of the cohort.

Presence of IBP by Assessment in Spondyloarthritis International Society criteria correlated with the prevalence of active sacroiliitis $(\mathrm{p}=0.024)$ and $\mathrm{SpA}$ (axial/SIJ; $\mathrm{p}=0.003$ ). The extent of psoriasis severity (PASI) correlated with both SIJ and whole spine SpA changes ( $p=0.02$ for both). Sex differences or biologic therapy did not affect the prevalence of SIJ or spine involvement.

Active and structural sacroiliitis was more prevalent compared to typical spinal SpA changes in the presented cohort. In particular, there were few SpA changes in the cervical spine. The most prominent axial findings included fatty corners and syndesmophytes. IBP presence and extensive skin disease correlated with SpA axial and SIJ changes.

Treating psoriatic arthritis to target: comorbidities, non- adherence, and factors related to the public health system prevent escalation of therapy in real life (Manoela F. Ferreira, Porto Alegre-RS, Brazil)

A treat-to-target (T2T) strategy in psoriatic arthritis (PsA) results in better functional and activity measures compared to standard care. Yet the implementation of a T2T strategy in daily practice is often limited. Dr. Ferreira conducted an observational retrospective cohort study to determine the prevalence of patients achieving minimal disease activity (MDA) in a PsA clinic based at a public university hospital in Brazil and analyzed the factors that prevent the implementation of a T2T strategy.

Medical records of 74 patients with PsA (40 women, 54\%) with 131 visits were reviewed from June 2016 through February 2017. The mean patient age was $57.9 \pm 10.9$ years, and the mean disease duration was $11.9 \pm 9.5$ years. Twenty-nine patients (39.2\%) had current or previous use of biological therapy. MDA score data were available for 113 visits (86.2\%), and MDA was achieved in 35 visits (31.0\%). Twenty-five (36.2\%) of the patients achieved MDA in at least 1 visit during followup. Although MDA was not achieved in $78(69.0 \%)$ visits, optimization of therapy was done in only $33(42.3 \%)$ of those visits. The main reasons treatment escalation was prevented were physician impression of remission $(n=24,55.3 \%)$, physician impression of pain and global components of MDA overestimated by comorbid conditions [i.e., fibromyalgia, osteoarthritis, soft tissue disease, and PsA deformities ( $\mathrm{n}=16,35.5 \%)$ ], nonadherence to previous prescriptions $(\mathrm{n}=8,17.8 \%)$, delay in receiving drugs from public health insurance $(\mathrm{n}=8,17.8 \%)$, adverse events $(\mathrm{n}=5,11.1 \%)$, patient low cognitive level $(\mathrm{n}=3$, $6.7 \%)$, and patient refusal to escalate therapy $(\mathrm{n}=2,4.4 \%)$. When a physician-determined impression of remission was recorded, the Psoriasis Area and Severity Index (PASI) and swollen joint components of the MDA were reached in almost $90 \%$ of visits $(n=21,87.5 \%)$.

The author concluded that rheumatologists were reluctant to escalate therapy in PsA even if patients were not in MDA if "objective" components of the MDA score, such as PASI and swollen joint count, were reached. Comorbid conditions, patient nonadherence to therapy, and factors related to the public health system influenced a tight control strategy in a real-life clinical practice.

Achieving pain visual analog scale target is the most difficult in patients with psoriatic arthritis (Flora Farkas, Dublin, Ireland)

Remission, defined by the minimal disease activity (MDA) criteria as MDA $\geq 5 / 7$, or a more stringent definition of very low disease activity (VLDA) 7/7, is considered an appropriate therapeutic target in psoriatic arthritis (PsA). Dr. Farkas performed a study to identify which disease component targets of PsA were the most difficult to achieve.

A total of 258 patients with PsA and 431 visits were

Personal non-commercial use only. The Journal of Rheumatology Copyright (C) 2018. All rights reserved. 
included in the analysis. MDA $\geq 5 / 7$ was achieved in 199 visits (46.2\%). Of those in $\mathrm{MDA} \geq 5 / 7$, there were 147 visits (73.9\%) that also achieved LDA (defined as MDA 5-6/7) and $52(26.1 \%)$ that achieved VLDA. The percentage of visits in which patients achieved the targets of tender joint count $\leq 1 / 68$, Psoriasis Area and Severity Index $\leq 1$, or body surface area $\leq 3 \%$, patient's global assessment activity visual analog scale (VAS) $\leq 2 \mathrm{~cm}$, pain VAS $\leq 1.5 \mathrm{~cm}$, and Health Assessment Questionnaire $\leq 0.5$ was significantly lower in LDA compared to the VLDA group, but there were no differences for the other MDA components. Patients fulfilled the pain VAS $\leq 1.5 \mathrm{~cm}$ target in only $26.3 \%$ of visits where they met MDA 5/7, 61.2\% with MDA 6/7, and 42.2\% with LDA 5-6/7. Logistic regression analysis revealed that the pain VAS target had the strongest correlation with not achieving a VLDA state. The study identified that the remission target of pain VAS $\leq 1.5 \mathrm{~cm}$ was the most difficult to achieve.

$G M-C S F+I F N-\gamma+C D 4+$ and $C D 8+T$ cell are enriched in synovial fluid of patients with psoriatic arthritis, while this subset is reduced in patient peripheral blood relative to healthy donors (Carmel Strober, Cambridge, UK)

Genetic studies have identified haplotypes containing IL23R, ILI2B, STAT3, and CARD9 variants that are associated with psoriasis and ankylosing spondylitis, and all function in the interleukin 23 (IL-23)/Th17 signaling pathway. In murine experimental autoimmune encephalomyelitis, IL-23 increases the pathogenicity of Th17 cells by upregulating granulocyte-macrophage colony-stimulating factor (GM-CSF) release, whereas IL-17 is not required for central nervous system inflammation. Dr. Strober investigated whether GM-CSF may be upregulated in psoriatic arthritis (PsA).

Study results revealed that in PsA synovial fluid, CD4+ T cells were significantly enriched with GM-CSF+ interferon- $\gamma$ $(\mathrm{IFN}-\gamma)+$ cells compared to peripheral blood (PB) CD4+ T cells $(\mathrm{p}<0.0001)$. There were significantly lower proportions of GM-CSF+IFN- $\gamma+$ CD4+ and CD8+ cells in PsA patients' PB relative to healthy donors $(\mathrm{p}<0.05)$. CD161 and IL-23R expression levels were comparable in GM-CSF+IFN- $\gamma+$ and GM-CSF+ cells, whereas CCR6 was lower for GMCSF+IFN- $\gamma+$ cells $(p<0.01)$. GM-CSF release was higher and IFN- $\gamma$ similar in patients with PsA relative to healthy donors. IL-17 release was increased ( $<<0.001)$, but GM-CSF downregulated by exogenous IL-23 ( $p<0.05)$. IL-23 also increased IL-17 $(\mathrm{p}<0.05)$ and decreased GM-CSF $(\mathrm{p}<0.001)$ using naive $\mathrm{CD} 4+$ cells differentiated in Th17-expanding conditions, and this was augmented by the addition of transforming growth factor- $\beta$. The study demonstrated co-expression of GM-CSF with IFN- $\gamma$ in diseased joints, but not in $\mathrm{PB}$ of patients with PsA. The mechanism GM-CSF+IFN- $\gamma+$ upregulation in PsA joints warrants further investigation.

Interleukin 17 blockade, but not tumor necrosis factor- $\alpha$ inhibition, alters the gut microbiota in psoriatic arthritis (Julia Manasson, New York, New York, USA)

Dr. Manasson presented preliminary results from a study that evaluated the effects biologic therapies have on the intestinal microbiome. Subjects with psoriatic arthritis who received interleukin 17 (IL-17) inhibitors demonstrated a microbial dysbiosis compared to those who received tumor necrosis factor- $\alpha$ inhibitors. Similar perturbations were seen in wild-type mice exposed to anti-IL-17 antibody.

\section{Poster Presentations}

Lenno Anjos (Sao Paulo, Brazil) characterized the rates of nonalcoholic fatty liver disease (NAFLD) in psoriatic arthritis (PsA). A total of 114 adults with PsA (64 men, 56\%) meeting the Classification Criteria for Psoriatic Arthritis (CASPAR) were evaluated (those with hepatitis $\mathrm{C}$ and significant alcohol intake were excluded). NAFLD was defined using abdominal ultrasound. The study population had a median age of 58 years and a median body mass index (BMI) of $31 \mathrm{~kg} / \mathrm{m}^{2}$. Of the subjects, $71 \%$ had polyarticular disease, $37 \%$ had axial disease, $16 \%$ had enthesitis, and 14\% had dactylitis; $64 \%$ were found to have NAFLD, which also correlated with higher average BMI $(\mathrm{p}<0.001)$, higher rates of dyslipidemia $(\mathrm{p}=0.002)$, and lower rates of dactylitis $(\mathrm{p}=0.003)$. These differences were maintained in the multivariate logistic regression. The author concluded that NAFLD is common in the PsA population and should be recognized by healthcare providers.

Sardar Bahadur (London, UK) used a novel hand radiograph scoring system to differentiate PsA from nodal osteoarthritis (NOA). The scoring system was based on interphalangeal joint, soft tissue, and bone features. The tests were based on radiographs collected from 48 subjects with PsA, 50 with NOA, and 1 with rheumatoid arthritis. Foot radiographs were also assessed. A musculoskeletal radiologist blinded to the clinical diagnoses identified 5 sets of normal hand radiographs and correctly identified the remainder of subjects using the scoring system. Rheumatology and radiology trainees correctly diagnosed $67 \%$ and $70 \%$ of radiographs, respectively. Foot radiographs showed fewer changes attributable to clinical disease. The author concluded that this novel scoring system appears to be effective at differentiating PsA from NOA and can be successfully taught to trainees and clinicians.

Peter Barnes (Portland, Oregon, USA) performed a retrospective analysis of patients with psoriasis and PsA to determine whether earlier treatment with tumor necrosis factor inhibitor (TNFi) leads to better quality-of-life outcomes. There was a statistically significant association between improvement in the Perceived Quality of Life-12 (PQoL-12) scale and timeliness of treatment, whereby a delay in treatment by 1 day resulted in a $4.4 \times 10^{-4}$ change in PQoL-12 ( $\mathrm{p}=0.007)$. There were no statistically significant differences between the timing of TNFi initiation and the Routine Assessment of Patient Index Data 3, 12-Item Short

Personal non-commercial use only. The Journal of Rheumatology Copyright @ 2018. All rights reserved. 
Form Health Survey, or body surface area affected by psoriasis. The author concluded that earlier initiation of TNFi had an effect on some quality-of-life measures.

Bashaar Boyce (Bath, UK) analyzed working patients from the Long-term Outcomes in PsA II UK-based multicenter prospective observational study to determine the validity of the Work Productivity and Activity Index Specific Health Problem (WPAI-SHP) in PsA. Of the 229 working patients, $177(77.3 \%)$ had complete data available at baseline and 3-month followup. The mean age was 48 years and the median disease duration was 6 years [interquartile range (IQR) 2-12]. The mean change of presenteeism was -7.9 (SD 30.0 ), standard error of the mean 16.2, and standardized response mean -0.26 . Among 105 patients $(59.3 \%)$ who reported no change between baseline and followup, the mean change in WPAI was absenteeism 2.9 (SD 30.7), presenteeism 4 (26.5), productivity loss 7.7 (27.2), and activity impairment 1.9 (24.4). Using the health-based anchor method (mean change in score among 72 patients who reported improvement), the minimally important difference (MID) for improvement was absenteeism -6.1 (26.2), presenteeism -20.8 (28.2), productivity loss -21.7 (30.8), and activity impairment -27.2 (29.5). Using the receiver-operation characteristic curve method, the MID was presenteeism -5.0 [area under the curve (AUC) 0.75], productivity loss -11.3 (AUC 0.76), and activity impairment -25.0 (AUC 0.79). The author concluded that presenteeism and productivity loss were moderately correlated with clinical measures, while responsiveness had a small to moderate correlation among the group as a whole.

Casandra Buzatu (Bucharest, Romania) performed a cross-sectional study to estimate the prevalence of hyperuricemia and identify associated factors in patients with PsA. The study included a total of 120 patients with PsA (69 women, $57.5 \%$ ), with a mean age of $54 \pm 11.8$ years and a mean disease duration of $7 \pm 7.4$ years. The median tender joint count was 2 (range 0-22), the median swollen joint count (SJC) was 0 (range 0-9), 24 (20\%) had moderate/ severe psoriasis, and $30(25 \%)$ were treated with a biologic agent. Notably, 33 (27.5\%) of subjects with PsA had hyperuricemia (defined as uric acid $>6.8 \mathrm{mg} / \mathrm{dl}$ ), which was associated with obesity $(1.86 ; \mathrm{p}=0.03)$, diabetes $(4.95$; $\mathrm{p}=0.01)$, and ischemic heart disease ( $3.61 ; \mathrm{p}=0.05)$, but not skin psoriasis using multivariate analysis. The author concluded that hyperuricemia in PsA appears to correlate with metabolic syndrome rather than skin psoriasis.

Francisco Colombres (Tucuman, Argentina; poster presented by Alberto Berman) evaluated the work capacity and quality of life of patients with recent-onset PsA, defined as disease duration of less than 3 years. The study included 108 patients ( 57 men, $53 \%$ ) who met CASPAR criteria, with a mean age of $48.4 \pm 12.5$ years and a mean disease duration of $17.6 \pm 9.8$ months. Only $4(6.6 \%)$ were HLA-B27positive. Disease was characterized by the Bath Ankylosing
Spondylitis Disease Activity Index (BASDAI) of $4.81 \pm 2.66$, Bath Ankylosing Spondylitis Functional Index (BASFI) of $3.75 \pm 2.70$, Psoriatic Arthritis Quality of Life (PsAQoL) scale of $7.24 \pm 6.44$, Health Assessment Questionnaire (HAQ) of $0.72 \pm 0.61$, physician's global assessment (PGA) by visual numeric scale (VNS) of $3.76 \pm 2.33$, and pain assessment by VNS of $5.22 \pm 2.98$. The average number of work days lost because of PsA in the preceding 6 months was $8.6 \pm 32.1$, which was significantly associated with the presence of enthesitis, number of swollen joints, higher BASDAI and BASFI scores, higher PGA and pain assessments, and lower education level ( $p<0.0001$ for all). Five patients lost their jobs because of PsA. The linear regression model showed that lower PsAQoL significantly correlated with PGA $(\mathrm{p}<0.001)$ and pain $(\mathrm{p}<0.01)$. The author concluded that deterioration of work capacity was associated with disease activity variables and functional disability in this cohort of patients with early PsA.

Elena Generali (Milan, Italy) evaluated whether serum antibodies against human LL-37, a cationic protein that is overexpressed in psoriatic skin lesions, are involved in the pathogenesis of PsA. Samples from 35 patients with PsA meeting CASPAR criteria and 34 healthy controls were studied, with 32 subjects (94.1\%) also having skin psoriasis and $5(14.3 \%)$ also having axial disease. The median 28-joint Disease Activity Score (DAS28)-C-reactive protein (CRP) was 3.19 (IQR 2.78-3.94). Of the subjects, 10 (28.6\%) were taking methotrexate and $9(25.7 \%)$ were taking TNFi. Human (h) LL-37 was synthesized and levels of anti-hLL-37 IgM and $\mathrm{IgG}$ were determined using an ELISA developed by the research group. Anti-hLL-37 IgM antibodies were detected in 22 subjects with PsA (63\%) compared to $2(6 \%)$ controls ( $\mathrm{p}<0.001)$. From those PsA subjects who had IgM antibodies, 2 (9\%) were in remission according to DAS28-CRP, compared to $5(39 \%)$ who did not have IgM antibodies $(\mathrm{p}=0.036)$. The author concluded that this is the first report suggesting an association of anti-hLL-37 antibodies with PsA and correlation with disease activity.

Catherine Hughes (London, UK) looked at quality of life and function in patients with PsA who were deemed to have minimal disease activity (MDA) compared to those who were not. Quality of life was assessed using the EQ-5D, and function was assessed using the HAQ-Disability Index (HAQ-DI). A total of 129 subjects with PsA were analyzed, with 83 (64\%) not achieving MDA, 46 (36\%) achieving MDA, and 19 (15\%) achieving very low MDA (VL-MDA). The mean DAS28 scores were $3.88 \pm 1.25$ for non-MDA subjects, $1.86 \pm 0.54$ for MDA subjects, and $1.6 \pm 0.46$ for VL-MDA subjects. Of the patients who achieved MDA, 20\% had an EQ-5D of 1 (which indicated perfect health), and 39\% had a HAQ-DI of 0 . The author concluded that disease state is an important factor for predicting quality-of-life outcomes.

Natsumi Ikumi (Dublin, Ireland) compared the different composite measures of MDA in patients with PsA, including

Personal non-commercial use only. The Journal of Rheumatology Copyright (C) 2018. All rights reserved. 
Disease Activity in Psoriatic Arthritis (DAPSA), clinical DAPSA (cDAPSA), Composite Psoriatic Disease Activity Index (CPDAI), and MDA. Cutoff points for remission were DAPSA $\leq 4$, cDAPSA $\leq 4$, CPDAI $\leq 2$, and MDA 7/7. Quality of life was assessed by the PsAQoL. A total of 258 patients with PsA who met CASPAR criteria and 435 visits were evaluated. The average number of patient visits that achieved remission were 68 according to DAPSA, 122 according to cDAPSA, 144 according to CPDAI, and 52 according to MDA 52. Interestingly, pain visual analog scale (VAS) and patient's global assessment questionnaires had higher values in those achieving CPDAI remission compared to the other composite measures $(p<0.01)$. There were no significant differences in PsAQoL in any of the measures. DAPSA and cDAPSA correlated strongly with MDA (Pearson coefficient $\mathrm{r}=0.7$ for both pairings). CPDAI did not correlate with DAPSA and weakly correlated with cDAPSA $(r=0.14)$ and $\operatorname{MDA}(r=0.2)$. The author concluded that MDA was the most stringent and CPDAI the least stringent remission measures. However, none of the measures predicted PsAQoL.

Anna Keszegpal (Leeds, UK) used a novel modified tape-stripping method to identify disease subtypes of psoriatic skin and predict treatment response. CuDerm adhesive tapes were used to remove the epidermis. Proteins were subsequently isolated and analyzed to quantify cytokines and chemokines. An example of this approach was presented in the context of a severely ill 38-year-old female patient who was hospitalized for generalized pustular psoriasis. Ten tapes were obtained from her lesional skin before and 5 days after the initiation of systemic therapy with prednisone, infliximab, and acitretin. Before therapy there were high levels of interleukin (IL)- $1 \beta$ (3.34 pg/ $\mu \mathrm{g}$ compared to an average of $0.58 \mathrm{pg} / \mu \mathrm{g}$ in plaque psoriasis) and IL-8 (104.67 $\mathrm{pg} / \mu \mathrm{g}$ compared to an average of $17.44 \mathrm{pg} / \mu \mathrm{g}$ in plaque psoriasis). These levels dramatically decreased with therapy. Other inflammatory markers, including S100A8/A9, CCL20, and GRO- $\alpha$, were also elevated, but did not change with therapy. IL-18 expression actually increased post-therapy. The author concluded that tape stripping appears to be a promising diagnostic method to analyze epidermal inflammation with the potential to identify molecular disease subgroups and choose individualized therapy.

Fabiana B. Oliveira (Porto Alegre-RS, Brazil) reported on 4 years of multidisciplinary management of patients with psoriasis and PsA in an outpatient clinic by dermatology-rheumatology teams. The study evaluated 84 patients (46 women, $54.76 \%$ ) with a mean age of $53.42 \pm 12.18$ years. A total of 162 consults were performed $(52.2 \%$ were first-time consults). The most common reason for an appointment in the clinic was joint symptoms $(n=57$, $67.9 \%)$. PsA was newly diagnosed in $19(22.6 \%)$ and confirmed in 21 patients $(25 \%)$, while $31(36.6 \%)$ were diagnosed with other rheumatic diseases. The preexisting diagnoses were modified in 18 patients $(21.4 \%)$ and treat- ments changed in 49 (53.3\%). The author concluded that a multidisciplinary approach with collaborative dermatology-rheumatology teams improves the diagnosis and management of psoriatic disease.

Perez-Alamino Rodolfo (Tucuman, Argentina) analyzed the prevalence of subclinical atherosclerosis in psoriatic disease in an Argentinian cohort. The study included 32 psoriasis subjects, 33 PsA subjects meeting CASPAR criteria, and 42 healthy controls. The psoriatic subjects (30 women, $46 \%$ ) had a mean age of $53.2 \pm 14.9$ years. The mean disease duration in patients with psoriasis was $17.8 \pm 13.6$ years versus 5.47 \pm 3.6 years in PsA. The psoriatic disease subjects had an average BMI of $32.8 \pm 6.4$, average glucose of 101.9 $\pm 25.3 \mathrm{mg} / \mathrm{dl}$, average Psoriasis Area and Severity Index (PASI) of $13.1 \pm 8.1$, and average DAS28 of $3.2 \pm 0.8$. Fifty-six subjects $(86.1 \%)$ were taking disease-modifying antirheumatic drugs (DMARD) and 22 (33.8\%) were taking biologic agents. Psoriatic patients had, on average, greater mean carotid artery intima-media thickness (IMT) compared to healthy controls $(0.68 \pm 0.24 \mathrm{~mm}$ psoriatic disease vs 0.49 $\pm 0.16 \mathrm{~mm}$ healthy; $\mathrm{p}=0.003$ ). No significant differences were found between psoriasis and PsA subjects. Atherosclerotic plaques were found in $9(14 \%)$ subjects with psoriatic disease, which was similar to healthy controls. IMT values did not correlate with various measures of disease activity. The author concluded that subjects with psoriatic disease had a higher prevalence of macrovascular disease compared to healthy controls.

Ari Polachek (Toronto, Canada) used a questionnaire-based approach to analyze the effect of PsA on fertility and pregnancy outcomes. Subjects who were diagnosed with PsA before at least 1 pregnancy were compared to healthy controls. A total of 74 PsA and 74 healthy control subjects were studied. There were no significant differences in age, ethnicity, marital status, level of education, number of pregnancies, and number of children between the 2 groups. There were no statistically significant differences in fertility between PsA and control subjects, including diagnosis of infertility [30\% PsA vs 22\% control; $\mathrm{p}=$ not significant (ns)], failure to conceive in 1 year (25\% PsA vs $15 \%$ control; $\mathrm{p}=\mathrm{ns})$, and use of fertility medications (5\% PsA vs $1 \%$ control; $\mathrm{p}=\mathrm{ns})$. The pregnancy outcomes were also similar in both groups, including live births (76\% PsA vs $76 \%$ control; $\mathrm{p}=\mathrm{ns}$ ), vaginal delivery ( $48 \%$ PsA vs $51 \%$ control; $\mathrm{p}=\mathrm{ns}$ ), gestational age (38.5 weeks PsA vs 38.3 weeks control; $\mathrm{p}=\mathrm{ns}$ ), birth weight ( $3.4 \mathrm{~kg}$ PsA vs $3.4 \mathrm{~kg}$ control; $\mathrm{p}=\mathrm{ns}$ ), rate of maternal and fetal complications, and the duration and rate of breastfeeding. Notably, $58 \%$ of cases reported favorable PsA disease activity (defined as no activity or improvement in disease) during pregnancy, though $50 \%$ subsequently had worsening disease activity within the first postpartum year. Similarly, $49 \%$ had favorable psoriasis disease activity during pregnancy, with $29 \%$ reporting worsening activity within the first postpartum year. The

Personal non-commercial use only. The Journal of Rheumatology Copyright @ 2018 . All rights reserved. 
author concluded that patients with PsA have similar fertility and pregnancy outcomes compared to healthy controls.

Reason Wilken (Sacramento, California, USA) characterized the glycosylation profiles (serum glycome and cell-surface glycome) of psoriasis and healthy subjects using a mass spectrometry-based multiple reaction monitoring method. The study analyzed 25 patients with psoriasis and 25 age- and sex-matched healthy controls, and 61 unique serum glycoproteins were identified that were differentially expressed in patients with psoriasis. Of these, 12 glycoconjugates could be used to distinguish disease state, and 34 glycoconjugates were differentially expressed following therapy with adalimumab, several of which were modifiers of lipoproteins. In addition, 20 glycoconjugates were found to correlate with psoriasis severity as measured by the PASI. The author concluded that glycans are likely contributors to the pathogenesis of psoriasis and may serve as biomarkers for presence of disease.

Alper Sari (Ankara, Turkey) characterized the clinical features, disease activity, and treatment choices for patients with PsA in a nationwide Turkish PsA registry (PsArt), comparing early-onset and late-onset PsA. The early-onset PsA group $(<60 \mathrm{yrs}$ of age at diagnosis) included 911 subjects, whereas the late-onset PsA group ( $\geq 60$ yrs of age at diagnosis) included 88 subjects. The mean age was $67.6 \pm 5.3$ years in the late-onset group compared to $44.9 \pm 11.4$ in the early-onset group. Mean disease duration was $2.7 \pm 3.2$ years in the late-onset group compared to $6.0 \pm 7.0$ years in the early-onset group ( $\mathrm{p}<0.001)$. Axial involvement was less frequent in the late-onset group (22.7\% late vs $36.3 \%$ early; $\mathrm{p}=0.01$ ), though the median BASFI score was higher (34 late vs 20 early; $\mathrm{p}=0.007$ ). Patients with late-onset disease appeared to have higher disease activity as demonstrated by mean tender joint count ( 3 late vs 2 early; $p=0.004$ ), mean SJC ( 1 late vs 0 early; $\mathrm{p}=0.04$ ), PGA (40 late vs 30 early; $\mathrm{p}=0.04$ ), pain VAS (50 late vs 40 early; $p=0.03$ ), and erythrocyte sedimentation rate $(29 \mathrm{~mm} / \mathrm{h}$ late vs $20 \mathrm{~mm} / \mathrm{h}$ early; $\mathrm{p}=0.001)$. However, TNFi use was significantly lower in the late-onset group (15.7\% late vs $34.7 \%$ early; $p=0.001$ ). The author concluded that there were clinical differences between late-onset and early-onset PsA. Even though patients with late-onset PsA appeared to have more active disease, biologic therapies were less frequently used, which could be due to clinician concern for safety in the face of other comorbidities.

Danielle Tartar (Sacramento, California, USA) performed Hi-D multicolor fluorescence activated cell sorting (FACS) analysis at the single-cell level to characterize $\mathrm{T}$ cells in patients with PsA compared to age- and sex-matched healthy controls. PB mononuclear cells (PBMC) and synovial fluid mononuclear cells (SFMC) were collected from 10 patients with PsA and 15 healthy controls. rIL-23 induced activated IL-17+ T cells were generated and FACS was performed to identify activated memory effector CD11a+CD45RO+IL-17+ $\mathrm{T}$ cells. Analysis demonstrated that IL-23 increased the percentage of IL-17+ CD3+ T cells in the PBMC and SFMC populations in both patients with PsA and healthy controls, yet levels of IL-17+ CD3+ T cells were higher in patients with PsA compared to controls $(\mathrm{p}<0.001)$. Patients with PsA demonstrated the following T cell phenotypes: $\alpha \beta$ T cells $(\mathrm{CD} 3+\alpha \beta \mathrm{TCR}+), \gamma \delta \mathrm{T}$ cells $(\mathrm{CD} 3+\gamma \delta \mathrm{TCR}+)$, mucosal associated invariant $\mathrm{T}$ (MAIT) cells (CD3+Va7.2TCR+ CD161high), and natural killer $\mathrm{T}$ cells (CD1d/PBS-57 tetramer+CD3+). The dominant phenotype was $\alpha \beta$ T cells (81.4 $\pm 2.8 \%$ in PBMC, $79.2 \pm 0.9 \%$ in SFMC). MAIT cells were enriched in SFMC $(3.3 \pm 0.7 \%)$ compared to PBMC (1.2 $\pm 0.1 \%$ ) cell populations and were predominantly CD8+. The author concluded that pathologic CD11a+CD45RO+IL-17+ $\mathrm{T}$ cells in PsA were composed of cells from both the innate and acquired immune system, which were dominated by the conventional Th17 $\alpha \beta$ T cells.

Cagri Unal (Istanbul, Turkey) investigated the occurrence of neuropathic pain in PsA and how it associates with disease activity, sleep, fatigue, and quality of life. The study included 40 patients with PsA (27 women, $67.5 \%$ ) who met CASPAR criteria, with a mean age of $50 \pm 9.8$ years and a mean disease duration of $99.7 \pm 97.7$ months. Mean DAS28 was $2.8 \pm 1.2$. The PainDETECT tool was used to identify neuropathic pain. Ambiguous neuropathic pain was identified in $4(10 \%)$ subjects, and $12(30 \%)$ had a PainDETECT score $>18$. PsAQoL and the Pittsburgh Sleep Quality Index (PSQI) were significantly higher in patients with neuropathic pain compared to those without ( $<<0.05)$. Further, PainDETECT correlated with several functional variables, including the PsAQoL (Spearman correlation $\varrho=0.66, p=0.0001$ ), PSQI $(\rho=0.40, p=0.01)$, and Multidimensional Assessment of Fatigue (MAF; $\rho=0.39, p=0.01$ ). In addition, neuropathic pain became more likely with increasing age $(\rho=0.40$, $\mathrm{p}=0.01)$. The author concluded that a substantial number of patients with PsA have neuropathic pain, which appears to correlate with worse quality of life and sleep disturbances.

Firat Ulutatar (Istanbul, Turkey) analyzed how fibromyalgia (FM) affects PsA disease activity and functional status. A total of 40 patients with PsA (27 women, 67.5\%) who met CASPAR criteria were enrolled in the study, with a mean age of $50 \pm 9.8$ years. Of the patients, 29 (72.5\%) fulfilled 2010 American College of Rheumatology criteria for FM. Patients with FM had higher mean Maastricht Ankylosing Spondylitis Enthesitis Score (MASES), PSQI, MAF, and PsAQoL ( $p<0.001)$. Further, Fibromyalgia Impact Questionnaire (FIQ) scores correlated with MASES (Spearman correlation $\rho=0.44, \mathrm{p}=0.01)$, MAF $(\mathrm{Q}=0.47$, $p=0.01)$, and PsAQoL $(\rho=0.50, p=0.005)$. There was no significant correlation between FIQ and DAS28. The author concluded that FM in patients with PsA is associated with poor quality of life, sleep disturbance, and fatigue.

Leonieke J.J. van Mens (Amsterdam, the Netherlands) conducted a cross-sectional study to assess the current clinical practice of defining residual disease and the subsequent

Personal non-commercial use only. The Journal of Rheumatology Copyright (C) 2018. All rights reserved. 
treatment decisions made in PsA. Among 142 patients with PsA, two-thirds (90/142) had residual disease activity, with half of these patients having moderate to high disease activity according to the Clinical Disease Activity Index. Of the patients, $74 \%$ with residual disease activity were being treated with either DMARD monotherapy or a first TNFi. In $23 \%$ of patients with residual disease, treatment adjustment was initiated. Treatment changes were considered less frequent in those taking a second TNFi. No differences were seen in disease activity and demographics between those with or without a treatment adjustment. Judgment by the physician and/or patient rather than objective hurdles to treatment intensification drove the decisions not to modify therapy. The author concluded that a majority of patients with residual disease had no treatment adjustment, which was not explained by comorbidity profiles or lack of treatment options.

Kim Wervers (Rotterdam, the Netherlands) described the effect of specific manifestations of PsA on health-related quality of life (HRQOL) in newly diagnosed patients. Data were collected at the time of PsA diagnosis on 399 patients (200 male, 50\%), with a mean age of $50.2 \pm 13.8$ years from the Dutch South-West Psoriatic Arthritis Registry. HRQOL was assessed by 8 domains of the Medical Outcomes Study Short Form-36 (SF-36) questionnaire. The majority of patients $(n=317)$ first presented with peripheral arthritis, whereas other subtypes of disease were infrequent at initial presentation, including enthesitis $(\mathrm{n}=34)$, axial disease $(\mathrm{n}=9)$, and dactylitis $(\mathrm{n}=39)$. Mean scores of SF-36 domains were lower than the Dutch reference population and similar across arthritis subtypes. Overall, the dactylitis subtype had higher SF-36 scores, and the enthesitis subtype had lower scores. Stratifying arthritis subtypes for the presence of enthesitis HRQOL decreased substantially for all groups with enthesitis across all domains. Severity of psoriasis and presence of dactylitis did not lead to significantly different SF-36 values compared to those who were not affected. Patients with chronic back pain had lower SF-36 scores, and no differences were found between those fulfilling Assessment of Spondyloarthritis international Society criteria for inflammatory back pain compared to those who did not. The author concluded that this study demonstrated a diminished HRQOL in PsA at the time of diagnosis compared to the Dutch reference population. The presence of enthesitis affected HRQOL more than the severity of joint involvement.

WanLi Zhou (Toronto, Ontario, Canada) examined the association between occupational-related mechanical factors and the severity of radiographic peripheral and axial joint damage in patients with longstanding PsA. A total of 307 patients with PsA ( $41.4 \%$ women) with a disease duration $\geq$ 10 years and an occupational history of paid occupations since age 18 were assessed for occupational-related mechanical exposures and physical activities. The mean age was $56.6 \pm$ 11.3 years, and the mean disease duration was $21 \pm 9.2$ years.
The mean duration in the workforce was $28.1 \pm 12.5$ years At the time of the study, $42.2 \%$ of the patients were working. Multivariable regression analysis showed that prolonged exposure to repetitive hand movements $(\beta=29.5, \mathrm{p}=0.007)$ and occupations that required a higher level of finger dexterity $(\beta=5.4, p=0.005)$ were associated with higher peripheral radiographic joint damage scores [by modified Steinbrocker Score (mSS)]. A borderline association was found between prolonged sitting time and lower $\mathrm{mSS}(\beta=-11.9, \mathrm{p}=0.085)$. With regard to axial damage, a borderline association was found between occupations that involved prolonged walking/running $(\beta=4.4, \mathrm{p}=0.04)$ and those that required a higher level of static strength $(\beta=0.6, p=0.052)$ and higher modified Stokes Ankylosing Spondylitis Spine Scores. The author concluded that a high level of occupation-related mechanical stress was associated with increased radiographic peripheral joint damage. This finding supports the potential role of microtrauma in the pathogenesis of PsA.

The following abstracts were presented at the conference, but are not published here because of confidentiality of the work in progress. Mariana Araujo Barbosa (Rio de Janeiro, Brazil) presented the clinical investigation of fissured tongue in psoriatic patients. Claudia Camargo (Rio de Janeiro, Brazil) introduced the investigation of an immunogenetic association between geographic tongue and psoriasis through frequencies of HLA and KIR genes in a mixed population. Daiane Matana (Rio de Janeiro, Brazil) evaluated bone mineral density in patients with PsA. Pedro Secchin (Rio de Janeiro, Brazil) compared the histological Trozak's score before and after phototherapy in treatment of psoriasis.

GRAPPA members were enthusiastic in their appreciation of the trainees' work and encouraged them to continue their research. The next GRAPPA Trainees Symposium will be held in July 2018 in Toronto, Canada.

\section{REFERENCES}

1. Coates LC, Ritchlin CT. GRAPPA Trainees Symposium 2009: a report from the GRAPPA 2009 annual meeting. J Rheumatol 2011;38:526-9.

2. Ritchlin CT. GRAPPA Trainees Symposium 2010: a report from the GRAPPA 2010 annual meeting. J Rheumatol 2012;39:394-7.

3. Ash Z, Ritchlin CT. GRAPPA Trainees Symposium 2011: a report from the GRAPPA 2011 annual meeting. J Rheumatol 2012;39:2184-8.

4. Garg N, Touma Z, Ritchlin CT. GRAPPA Trainees Symposium 2012: a report from the GRAPPA 2012 annual meeting. J Rheumatol 2013;40:1413-8.

5. Szentpetery A, Johnson MA, Ritchlin CT. GRAPPA Trainees Symposium 2013: a report from the GRAPPA 2013 annual meeting. J Rheumatol 2014;41:1200-5.

6. Eder L, Tillett W, Ritchlin CT. GRAPPA Trainees Symposium 2014: a report from the GRAPPA 2014 Annual Meeting. J Rheumatol 2015;42:1016-20.

7. Milliken M, Generali E, Marin J, Ritchlin CT. GRAPPA Trainees Symposium 2015: a report from the GRAPPA 2015 annual meeting. J Rheumatol 2016;43:952-8 